\title{
A New Proof of the Jawerth-Franke Embedding
}

\author{
Jan VYBíRAL
}

\author{
Mathematisches Institut \\ Friedrich-Schiller-Universität Jena \\ Ernst-Abbe-Platz 3 \\ 07740 Jena - Germany \\ vybiral@minet.uni-jena.de
}

Received: May 28, 2007

Accepted: July 2, 2007

\section{ABSTRACT}

We present an alternative proof of the Jawerth embedding

$$
F_{p_{0} q}^{s_{0}}\left(\mathbb{R}^{n}\right) \longleftrightarrow B_{p_{1} p_{0}}^{s_{1}}\left(\mathbb{R}^{n}\right)
$$

where

$$
-\infty<s_{1}<s_{0}<\infty, \quad 0<p_{0}<p_{1} \leq \infty, \quad 0<q \leq \infty
$$

and

$$
s_{0}-\frac{n}{p_{0}}=s_{1}-\frac{n}{p_{1}} .
$$

The original proof given in [3] uses interpolation theory. Our proof relies on wavelet decompositions and transfers the problem from function spaces to sequence spaces. Using similar techniques, we also recover the embedding of Franke [2].

Key words: Besov spaces, Triebel-Lizorkin spaces, Sobolev embedding, Jawerth-Franke embedding.

2000 Mathematics Subject Classification: 46E35. 


\section{Introduction}

Let $B_{p q}^{s}\left(\mathbb{R}^{n}\right)$ and $F_{p q}^{s}\left(\mathbb{R}^{n}\right)$ denote the Besov and Triebel-Lizorkin function spaces, respectively. The classical Sobolev embedding theorem can be extended to these two scales.

Theorem 0.1. Let $-\infty<s_{1}<s_{0}<\infty$ and $0<p_{0}<p_{1} \leq \infty$ with

$$
s_{0}-\frac{n}{p_{0}}=s_{1}-\frac{n}{p_{1}} .
$$

(i) If $0<q_{0} \leq q_{1} \leq \infty$, then

$$
B_{p_{0} q_{0}}^{s_{0}}\left(\mathbb{R}^{n}\right) \longleftrightarrow B_{p_{1} q_{1}}^{s_{1}}\left(\mathbb{R}^{n}\right) .
$$

(ii) If $0<q_{0}, q_{1} \leq \infty$ and $p_{1}<\infty$, then

$$
F_{p_{0} q_{0}}^{s_{0}}\left(\mathbb{R}^{n}\right) \longleftrightarrow F_{p_{1} q_{1}}^{s_{1}}\left(\mathbb{R}^{n}\right) .
$$

We observe that there is no condition on the fine paramters $q_{0}, q_{1}$ in (2). This surprising effect was first observed in full generality by Jawerth, [3]. Using (2), we may prove

and

$$
F_{p_{0} q}^{s_{0}}\left(\mathbb{R}^{n}\right) \longleftrightarrow F_{p_{1} p_{1}}^{s_{1}}\left(\mathbb{R}^{n}\right)=B_{p_{1} p_{1}}^{s_{1}}\left(\mathbb{R}^{n}\right)
$$

$$
B_{p_{0} p_{0}}^{s_{0}}\left(\mathbb{R}^{n}\right)=F_{p_{0} p_{0}}^{s_{0}}\left(\mathbb{R}^{n}\right) \longleftrightarrow F_{p_{1} q}^{s_{1}}\left(\mathbb{R}^{n}\right)
$$

for every $0<q \leq \infty$. But Jawerth [3] and Franke [2] showed that these embeddings are not optimal and may be improved.

Theorem 0.2. Let $-\infty<s_{1}<s_{0}<\infty, 0<p_{0}<p_{1} \leq \infty$, and $0<q \leq \infty$ with (1).

(i) Then

$$
F_{p_{0} q}^{s_{0}}\left(\mathbb{R}^{n}\right) \longleftrightarrow B_{p_{1} p_{0}}^{s_{1}}\left(\mathbb{R}^{n}\right)
$$

(ii) If $p_{1}<\infty$, then

$$
B_{p_{0} p_{1}}^{s_{0}}\left(\mathbb{R}^{n}\right) \longleftrightarrow F_{p_{1} q}^{s_{1}}\left(\mathbb{R}^{n}\right)
$$

The original proofs (see $[2,3])$ use interpolation techniques. We rely on a different method. First, we observe that using (for example) the wavelet decomposition method, (3) and (4) are equivalent to

$$
f_{p_{0} q}^{s_{0}} \longleftrightarrow b_{p_{1} p_{0}}^{s_{1}} \quad \text { and } \quad b_{p_{0} p_{1}}^{s_{0}} \longleftrightarrow f_{p_{1} q}^{s_{1}}
$$


under the same restrictions on parameters $s_{0}, s_{1}, p_{0}, p_{1}, q$ as in Theorem 0.2. Here, $b_{p q}^{s}$ and $f_{p q}^{s}$ stands for the sequence spaces of Besov and Triebel-Lizorkin type. We prove (5) directly using the technique of non-increasing rearrangement on a rather elementary level.

All the unimportant constants are denoted by the letter $c$, whose meaning may differ from one occurrence to another. If $\left\{a_{n}\right\}_{n=1}^{\infty}$ and $\left\{b_{n}\right\}_{n=1}^{\infty}$ are two sequences of positive real numbers, we write $a_{n} \lesssim b_{n}$ if, and only if, there is a positive real number $c>0$ such that $a_{n} \leq c b_{n}, n \in \mathbb{N}$. Furthermore, $a_{n} \approx b_{n}$ means that $a_{n} \lesssim b_{n}$ and simultaneously $b_{n} \lesssim a_{n}$.

\section{Notation and definitions}

We introduce the sequence spaces associated with the Besov and Triebel-Lizrokin spaces. Let $m \in \mathbb{Z}^{n}$ and $\nu \in \mathbb{N}_{0}$. Then $Q_{\nu m}$ denotes the closed cube in $\mathbb{R}^{n}$ with sides parallel to the coordinate axes, centred at $2^{-\nu} m$, and with side length $2^{-\nu}$. By $\chi_{\nu m}=\chi_{Q_{\nu m}}$ we denote the characteristic function of $Q_{\nu m}$. If

$$
\lambda=\left\{\lambda_{\nu m}: \nu \in \mathbb{N}_{0}, m \in \mathbb{Z}^{n}\right\},
$$

$-\infty<s<\infty$, and $0<p, q \leq \infty$, we set

$$
\left\|\lambda \mid b_{p q}^{s}\right\|=\left(\sum_{\nu=0}^{\infty} 2^{\nu\left(s-\frac{n}{p}\right) q}\left(\sum_{m \in \mathbb{Z}^{n}}\left|\lambda_{\nu m}\right|^{p}\right)^{\frac{q}{p}}\right)^{\frac{1}{q}},
$$

appropriately modified if $p=\infty$ and/or $q=\infty$. If $p<\infty$, we define also

$$
\left\|\lambda\left|f_{p q}^{s}\|=\|\left(\sum_{\nu=0}^{\infty} \sum_{m \in \mathbb{Z}^{n}}\left|2^{\nu s} \lambda_{\nu m} \chi_{\nu m}(\cdot)\right|^{q}\right)^{1 / q}\right| L_{p}\left(\mathbb{R}^{n}\right)\right\| .
$$

The connection between the function spaces $B_{p q}^{s}\left(\mathbb{R}^{n}\right), F_{p q}^{s}\left(\mathbb{R}^{n}\right)$ and the sequence spaces $b_{p q}^{s}, f_{p q}^{s}$ may be given by various decomposition techniques, we refer to [7, chapters 2 and 3] for details and further references.

As a result of these characterizations, (3) is equivalent to (5).

We use the technique of non-increasing rearrangement. We refer to [1, chapter 2$]$ for details.

Definition 1.1. Let $\mu$ be the Lebesgue measure in $\mathbb{R}^{n}$. If $h$ is a measurable function on $\mathbb{R}^{n}$, we define the non-increasing rearrangement of $h$ through

$$
h^{*}(t)=\sup \left\{\lambda>0: \mu\left\{x \in \mathbb{R}^{n}:|h(x)|>\lambda\right\}>t\right\}, \quad t \in(0, \infty) .
$$

We denote its averages by

$$
h^{* *}(t)=\frac{1}{t} \int_{0}^{t} h^{*}(s) d s, \quad t>0 .
$$


We shall use the following properties. The first two are very well known and their proofs may be found in [1, Proposition 1.8 in chapter 2, Theorem 3.10 in chapter 3].

Lemma 1.2. If $0<p \leq \infty$, then

$$
\left\|h\left|L_{p}\left(\mathbb{R}^{n}\right)\|=\| h^{*}\right| L_{p}(0, \infty)\right\|
$$

for every measurable function $h$.

Lemma 1.3. If $1<p \leq \infty$, then there is a constant $c_{p}$ such that

$$
\left\|h^{* *}\left|L_{p}(0, \infty)\left\|\leq c_{p}\right\| h^{*}\right| L_{p}(0, \infty)\right\|
$$

for every measurable function $h$.

Lemma 1.4. Let $h_{1}$ and $h_{2}$ be two non-negative measurable functions on $\mathbb{R}^{n}$. If $1 \leq p \leq \infty$, then

$$
\left\|h_{1}+h_{2}\left|L_{p}\left(\mathbb{R}^{n}\right)\|\leq\| h_{1}^{*}+h_{2}^{*}\right| L_{p}(0, \infty)\right\| .
$$

Proof. The proof follows from Theorems 3.4 and 4.6 in [1, chapter2].

\section{Main results}

In this part, we present a direct proof of the discrete versions of Jawerth and Franke embedding. We start with the Jawerth embedding.

Theorem 2.1. Let $-\infty<s_{1}<s_{0}<\infty, 0<p_{0}<p_{1} \leq \infty$, and $0<q \leq \infty$. Then

$$
f_{p_{0} q}^{s_{0}} \longleftrightarrow b_{p_{1} p_{0}}^{s_{1}} \quad \text { if } \quad s_{0}-\frac{n}{p_{0}}=s_{1}-\frac{n}{p_{1}} .
$$

Proof. Using the elementary embedding

$$
f_{p q_{0}}^{s} \longleftrightarrow f_{p q_{1}}^{s} \text { if } \quad 0<q_{0} \leq q_{1} \leq \infty
$$

and the lifting property of Besov and Triebel-Lizorkin spaces (which is even simpler in the language of sequence spaces), we may restrict ourselves to the proof of

$$
f_{p_{0} \infty}^{s} \hookrightarrow b_{p_{1} p_{0}}^{0}, \quad \text { where } \quad s=n\left(\frac{1}{p_{0}}-\frac{1}{p_{1}}\right) .
$$

Let $\lambda \in f_{p_{0} \infty}^{s}$ and set

$$
h(x)=\sup _{\nu \in \mathbb{N}_{0}} 2^{\nu s} \sum_{m \in \mathbb{Z}^{n}}\left|\lambda_{\nu m}\right| \chi_{\nu m}(x) .
$$


Hence

$$
\left|\lambda_{\nu m}\right| \leq 2^{-\nu s} \inf _{x \in Q_{\nu m}} h(x), \quad \nu \in \mathbb{N}_{0}, \quad m \in \mathbb{Z}^{n} .
$$

Using this notation,

$$
\left\|\lambda\left|f_{p_{0} \infty}^{s}\|=\| h\right| L_{p_{0}}\left(\mathbb{R}^{n}\right)\right\|
$$

and

$$
\begin{aligned}
\left\|\lambda \mid b_{p_{1} p_{0}}^{0}\right\|^{p_{0}} & \leq \sum_{\nu=0}^{\infty} 2^{-\nu n}\left(\sum_{m \in \mathbb{Z}^{n}} \inf _{x \in Q_{\nu m}} h(x)^{p_{1}}\right)^{p_{0} / p_{1}} \\
& \leq \sum_{\nu=0}^{\infty} 2^{-\nu n}\left(\sum_{k=1}^{\infty} h^{*}\left(2^{-\nu n} k\right)^{p_{1}}\right)^{p_{0} / p_{1}} .
\end{aligned}
$$

Using the monotonicity of $h^{*}$ and $p_{0}<p_{1}$ we get

$$
\begin{aligned}
\left\|\lambda \mid b_{p_{1} p_{0}}^{0}\right\|^{p_{0}} & \lesssim \sum_{\nu=0}^{\infty} 2^{-\nu n}\left(\sum_{l=0}^{\infty} 2^{n l} \cdot\left(2^{n}-1\right) \cdot h^{*}\left(2^{-\nu n} 2^{n l}\right)^{p_{1}}\right)^{p_{0} / p_{1}} \\
& \lesssim \sum_{\nu=0}^{\infty} 2^{-\nu n} \sum_{l=0}^{\infty} 2^{n l \frac{p_{0}}{p_{1}}} h^{*}\left(2^{-\nu n} 2^{n l}\right)^{p_{0}} .
\end{aligned}
$$

We substitute $j=l-\nu$ and obtain

$$
\begin{aligned}
\left\|\lambda \mid b_{p_{1} p_{0}}^{0}\right\|^{p_{0}} & \lesssim \sum_{j=-\infty}^{\infty} \sum_{\nu=-j}^{\infty} 2^{-\nu n} 2^{n(\nu+j) \frac{p_{0}}{p_{1}}} h^{*}\left(2^{j n}\right)^{p_{0}} \\
& =\sum_{j=-\infty}^{\infty} 2^{n j \frac{p_{0}}{p_{1}}} h^{*}\left(2^{j n}\right)^{p_{0}} \sum_{\nu=-j}^{\infty} 2^{n \nu\left(\frac{p_{0}}{p_{1}}-1\right)} \\
& \approx \sum_{j=-\infty}^{\infty} 2^{n j} h^{*}\left(2^{n j}\right)^{p_{0}} \approx\left\|h^{*}\left|L_{p_{0}}(0, \infty)\left\|^{p_{0}}=\right\| h\right| L_{p_{0}}\left(\mathbb{R}^{n}\right)\right\|^{p_{0}}
\end{aligned}
$$

If $p_{1}=\infty$, only notational changes are necessary.

Theorem 2.2. Let $-\infty<s_{1}<s_{0}<\infty, 0<p_{0}<p_{1}<\infty$, and $0<q \leq \infty$. Then

$$
b_{p_{0} p_{1}}^{s_{0}} \longleftrightarrow f_{p_{1} q}^{s_{1}} \quad \text { if } \quad s_{0}-\frac{n}{p_{0}}=s_{1}-\frac{n}{p_{1}} .
$$

Proof. Using the lifting property and (6), we may suppose that $s_{1}=0$ and $0<q<p_{0}$.

By Lemma 1.4, we observe that

$$
\left\|\lambda\left|f_{p_{1} q}^{0}\|=\|\left(\sum_{\nu=0}^{\infty} \sum_{m \in \mathbb{Z}^{n}}\left|\lambda_{\nu m}\right|^{q} \chi_{\nu m}(x)\right)^{1 / q}\right| L_{p_{1}}\left(\mathbb{R}^{n}\right)\right\|
$$


may be estimated from above by

$$
\left\|\sum_{\nu=0}^{\infty} \sum_{m=0}^{\infty} \tilde{\lambda}_{\nu m}^{q} \tilde{\chi}_{\nu m}(\cdot) \mid L_{\frac{p_{1}}{q}}(0, \infty)\right\|^{1 / q}
$$

where $\tilde{\lambda}_{\nu}=\left\{\tilde{\lambda}_{\nu m}\right\}_{m=0}^{\infty}$ is a non-increasing rearrangement of $\lambda_{\nu}=\left\{\lambda_{\nu m}\right\}_{m \in \mathbb{Z}^{n}}$ and $\tilde{\chi}_{\nu m}$ is a characteristic function of the interval $\left(2^{-\nu n} m, 2^{-\nu n}(m+1)\right)$.

Using duality, (7) may be rewritten as

$$
\sup _{g}\left(\int_{0}^{\infty} g(x)\left(\sum_{\nu=0}^{\infty} \sum_{m=0}^{\infty} \tilde{\lambda}_{\nu m}^{q} \tilde{\chi}_{\nu m}(x)\right) d x\right)^{1 / q}=\sup _{g}\left(\sum_{\nu=0}^{\infty} \sum_{m=0}^{\infty} 2^{-\nu n} \tilde{\lambda}_{\nu m}^{q} g_{\nu m}\right)^{1 / q}
$$

where the supremum is taken over all non-increasing non-negative measurable functions $g$ with $\left\|g \mid L_{\beta}(0, \infty)\right\| \leq 1$ and $g_{\nu m}=2^{\nu n} \int g(x) \tilde{\chi}_{\nu m}(x) d x$. Here, $\beta$ is the conjugated index to $\frac{p_{1}}{q}$. Similarly, $\alpha$ stands for the conjugated index to $\frac{p_{0}}{q}$.

We use twice Hölder's inequality and estimate (8) from above by

$$
\left(\sum_{\nu=0}^{\infty} 2^{-\nu n}\left(\sum_{m=0}^{\infty} \tilde{\lambda}_{\nu m}^{p_{0}}\right)^{\frac{p_{1}}{p_{0}}}\right)^{1 / p_{1}} \cdot \sup _{g}\left(\sum_{\nu=0}^{\infty} 2^{-\nu n}\left(\sum_{m=0}^{\infty} g_{\nu m}^{\alpha}\right)^{\frac{\beta}{\alpha}}\right)^{\frac{1}{\beta q}}
$$

Since $s_{0}=n\left(\frac{1}{p_{0}}-\frac{1}{p_{1}}\right)$ and $p_{1}\left(s_{0}-\frac{n}{p_{0}}\right)=-n$, the first factor in (9) is equal to $\left\|\lambda \mid b_{p_{0} p_{1}}^{s_{0}}\right\|$. To finish the proof, we have to show that there is a number $c>0$ such that

$$
\left(\sum_{\nu=0}^{\infty} 2^{-\nu n}\left(\sum_{m=0}^{\infty} g_{\nu m}^{\alpha}\right)^{\frac{\beta}{\alpha}}\right)^{\frac{1}{\beta q}} \leq c
$$

holds for every non-increasing non-negative measurable functions $g$ with $\left\|g \mid L_{\beta}(0, \infty)\right\|$ $\leq 1$. We fix such a function $g$. Using the monotonicity of $g$, we get

$$
\begin{aligned}
& \sum_{m=0}^{\infty} g_{\nu m}^{\alpha}=\sum_{l=0}^{\infty} \sum_{m=2^{l n}-1}^{2^{(l+1) n}}\left(2^{\nu n} \int_{2^{-\nu n} m}^{2^{-\nu n}(m+1)} g(x) d x\right)^{\alpha} \\
& \lesssim \sum_{l=0}^{\infty} 2^{l n}\left(2^{\nu n} \int_{2^{-\nu n}\left(2^{l n}-1\right)}^{2^{-\nu n} 2^{l n}} g(x) d x\right)^{\alpha} \leq \sum_{l=0}^{\infty} 2^{l n}\left(g^{* *}\right)^{\alpha}\left(2^{(l-\nu) n}\right) .
\end{aligned}
$$


We use $1<\beta<\alpha$, Lemma 1.3 and obtain

$$
\begin{aligned}
\left(\sum_{\nu=0}^{\infty} 2^{-\nu n}\left(\sum_{m=0}^{\infty} g_{\nu m}^{\alpha}\right)^{\frac{\beta}{\alpha}}\right)^{1 / \beta} & \leq\left(\sum_{\nu=0}^{\infty} 2^{-\nu n}\left(\sum_{l=0}^{\infty} 2^{l n}\left(g^{* *}\right)^{\alpha}\left(2^{(l-\nu) n}\right)\right)^{\frac{\beta}{\alpha}}\right)^{1 / \beta} \\
& \leq\left(\sum_{\nu=0}^{\infty} 2^{-\nu n} \sum_{l=0}^{\infty} 2^{l n \frac{\beta}{\alpha}}\left(g^{* *}\right)^{\beta}\left(2^{(l-\nu) n}\right)\right)^{1 / \beta} \\
& \leq\left(\sum_{k=-\infty}^{\infty} 2^{k n \frac{\beta}{\alpha}} \sum_{\nu=-k}^{\infty} 2^{\nu n\left(\frac{\beta}{\alpha}-1\right)}\left(g^{* *}\right)^{\beta}\left(2^{k n}\right)\right)^{1 / \beta} \\
& \lesssim\left(\sum_{k=-\infty}^{\infty} 2^{k n}\left(g^{* *}\right)^{\beta}\left(2^{k n}\right)\right)^{1 / \beta} \\
& \lesssim\left\|g^{* *}\left|L_{\beta}(0, \infty)\|\leq c\| g\right| L_{\beta}(0, \infty)\right\| \leq c .
\end{aligned}
$$

Taking the $\frac{1}{q}$-power of this estimate, we finish the proof of (10).

The Theorems 2.1 and 2.2 are sharp in the following sense.

Theorem 2.3. Let $-\infty<s_{1}<s_{0}<\infty, 0<p_{0}<p_{1} \leq \infty$, and $0<q_{0}, q_{1} \leq \infty$ with

$$
s_{0}-\frac{n}{p_{0}}=s_{1}-\frac{n}{p_{1}} \text {. }
$$

(i) If

$$
f_{p_{0} q_{0}}^{s_{0}} \hookrightarrow b_{p_{1} q_{1}}^{s_{1}}
$$

then $q_{1} \geq p_{0}$.

(ii) If $p_{1}<\infty$ and

$$
b_{p_{0} q_{0}}^{s_{0}} \longleftrightarrow f_{p_{1} q_{1}}^{s_{1}},
$$

then $q_{0} \leq p_{1}$.

Remark 2.4. Using (any of) the usual decomposition techniques, the same statements hold true also for the function spaces. These results were first proved in [4].

Proof. (i) Suppose that $0<q_{1}<p_{0}<\infty$ and set

$$
\lambda_{\nu m}= \begin{cases}\nu^{-\frac{1}{q_{1}}} 2^{\nu\left(\frac{n}{p_{1}}-s_{1}\right)} & \text { if } \nu \in \mathbb{N} \text { and } m=0, \\ 0, & \text { otherwise. }\end{cases}
$$

A simple calculation shows that $\left\|\lambda \mid f_{p_{0} q_{0}}^{s_{0}}\right\|<\infty$ and $\left\|\lambda \mid b_{p_{1} q_{1}}^{s_{1}}\right\|=\infty$. Hence, (11) does not hold.

(ii) Suppose that $0<p_{1}<q_{0} \leq \infty$ and set 


$$
\lambda_{\nu m}= \begin{cases}\nu^{-\frac{1}{p_{1}}} 2^{\nu\left(\frac{n}{p_{1}}-s_{1}\right)} & \text { if } \nu \in \mathbb{N} \text { and } m=0, \\ 0, & \text { otherwise. }\end{cases}
$$

Again, it is a matter of simple calculation to show, that $\left\|\lambda \mid b_{p_{0} q_{0}}^{s_{0}}\right\|<\infty$ and $\left\|\lambda \mid f_{p_{1} q_{1}}^{s_{1}}\right\|=\infty$. Hence, (12) is not true.

\section{References}

[1] C. Bennett and R. Sharpley, Interpolation of operators, Pure and Applied Mathematics, vol. 129, Academic Press Inc., Boston, MA, 1988.

[2] J. Franke, On the spaces $\mathbf{F}_{p q}^{s}$ of Triebel-Lizorkin type: pointwise multipliers and spaces on domains, Math. Nachr. 125 (1986), 29-68.

[3] B. Jawerth, Some observations on Besov and Lizorkin-Triebel spaces, Math. Scand. 40 (1977), no. $1,94-104$.

[4] W. Sickel and H. Triebel, Hölder inequalities and sharp embeddings in function spaces of $B_{p q}^{s}$ and $F_{p q}^{s}$ type, Z. Anal. Anwendungen 14 (1995), no. 1, 105-140.

[5] H. Triebel, Theory of function spaces, Monographs in Mathematics, vol. 78, Birkhäuser Verlag, Basel, 1983.

[6] _ Theory of function spaces, II, Monographs in Mathematics, vol. 84, Birkhäuser Verlag, Basel, 1992.

[7] _ Theory of function spaces, III, Monographs in Mathematics, vol. 100, Birkhäuser Verlag, Basel, 2006. 